\title{
Short Paper: R\&D of a Dual mode acoustic modem testbed for shallow water channels
}

\author{
Feng TONG ${ }^{1,2}$ Shengyong Zhou ${ }^{1}$ \\ 1. Key Laboratory of Underwater Acoustic \\ communication and Marine Information Technology of \\ the MOE, Xiamen University, Xiamen, China \\ ftong@cs.ucsd.edu \\ Bridget Benson ${ }^{2}$, Ryan Kastner ${ }^{2}$ \\ 2. Department of Computer Science and Engineering \\ University of California San Diego, \\ San Diego, USA \\ kastner@cs.ucsd.edu
}

\begin{abstract}
Recent years witnessed an increasing requirement of marine missions like oceanographic investigation, environmental monitoring, underwater structure inspection and sea bottom resource exploitation, which urged the R\&D of high performance underwater acoustic (UWA) modems. However, as underwater acoustic channels pose multiple difficulties such as multipath, time-space selectivity, frequency dependent noise, and Doppler shifts on transmission, currently there is not a single modulation strategy that can provide high data rate, energy efficiency and channel robustness simultaneously. Enlightened by the similar dual mode (DM) systems developed in wireless channels, this paper presents the R\&D of a DM UWA modem testbed system, which enables the switch between DSSS and OFDM scheme to accommodate different channel situations. Experimental results obtained in a physical shallow water channel demonstrate the effectiveness of the proposed system.
\end{abstract}

\section{INTRODUCTION}

More and more interests for various marine missions such as environmental (pollution, coral reef, seismic, ocean current, etc.) monitoring, underwater structure inspection (oil platform, pipeline, undersea tunnel, etc.), oceanographic investigation, and sea bottom resource 
exploitation drive increasing attention on the R\&D of underwater acoustic (UWA)

communication modems. However, UWA channels present significant challenges for underwater acoustic communication ${ }^{[1-4],}$ which is recognized as much more difficult compared to counterparts in other media, such as the radio channel. Main obstacles for reliable high speed UWA communications through severely band-limited UWA channels consists of multipath, fading, Doppler effect and long propagation delay.

There is a great amount of literature on modulation strategies used to develop UWA communication system (such as MFSK, DSSS, FHSS, OFDM and QPSK), each of which can achieve satisfactory performance with respect to certain UWA channel. Nonetheless, considering the diversity of marine missions, there is a lack of a single modulation technology that can attain different performance requirements, i.e., high data rate, channel adaptability and high energy efficiency in different environments. Inspired by the dual mode systems developed for wireless channels that allow handsets to operate on different networks such as CDMA, GSM and Wi-Fi to meet different needs ${ }^{[5]}$, a dual mode UWA modem can provide a flexible solution to effectively operate under different kinds of environments. The WHOI micromodem $^{[6]}$ has dual mode of operation: high rate PSK to achieve bandwidth efficiency, as well as low rate FSK used for robust communication. In this paper we present the R\&D of a dual mode UWA modem testbed which can switch between DSSS and OFDM mode. Specifically, the proposed DM modem testbed can use the DSSS mode to achieve a reliable and low data rate link with low energy consumption and use the OFDM mode to enable high data rate communications when the channel is relatively ideal. The switch of different modulation modes is controlled by a mode selection mechanism according to the quality of the underwater channel, as well as the requirement of mission. 
Though both DSSS and OFDM systems have been widely investigated in several papers ${ }^{\text {[7-11] }}$ for underwater scenarios, there are few works that reported simultaneous adoption of both protocols in one system. In this paper, we present the system design, modulation modular, mode selection protocol, and DSP implementation, and report experimental results showing the dual mode system’s performance on a real UWA communication scenario.

The rest of the paper is organized as follows: Section II presents the basis of the dual mode system. Section III introduces the system design and sea experiment configuration. Section IV provides experimental results. We conclude in section $\mathrm{V}$.

\section{BASIS OF DUAL MODE MODEM}

A dual-mode phone is defined as a phone that uses more than one technique for sending and receiving voice and data. Similarly, a dual-mode UWA modem uses more than one modulation scheme for sending and receiving data. In the proposed DM system, DSSS and OFDM are adopted as the candidate modulation techniques and a mode selection protocol based on channel probing is employed to control switching between the two modes. As the main focus of this paper is the dual mode mechanism, we directly utilize classic DSSS and OFDM schemes in the design of the modulation and demodulation modulars.

\subsection{DSSS modular}

Firstly, let us consider the classic DS-DBPSK strategy. The baseband waveform can be expressed as:

$$
\begin{gathered}
b(t)=\sum_{n=-\infty}^{\infty} b_{n} g_{T_{b}}\left(t-n T_{b}\right)=\sum_{n=-\infty}^{\infty} b_{\mid t} g_{\left.T_{b}\right\rfloor}\left(t-n T_{b}\right) \\
\text { and PN sequence can be expressed as: } \\
c(t)=\sum_{k} c_{(k \bmod G)} g_{T_{e}}\left(k-T_{c}\right)=\sum_{k} c_{\left\lfloor\frac{1}{T_{e}}\right\rfloor_{\bmod G)} g_{T_{e}}\left(k-T_{c}\right)}
\end{gathered}
$$

after carrier modulation the transmission signal is produced as:

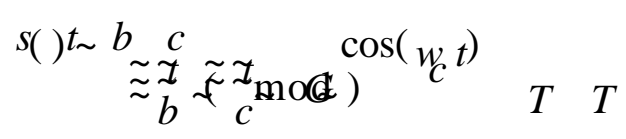


Where $b_{n}$ is the bits after differential coding, $T_{c}$ is the chip width, $g_{T b}$ is a rectanglar pulse with width $T b, g_{T C}$ is a rectangular pulse with width $T_{c}$, and $G$ is the spread spectrum gain. A block chart of the DSSS modular is shown in Fig. 1.

\subsection{OFDM modular}

For the OFDM scheme, the original bit is firstly mapped to the DQPSK constellation to form the sequence $\{d 0, \sim, d N-1\}$, where $d n=a n+\mathrm{j} b n$. The DQPSK sequence is further mapped to the frequency band associated with OFDM modulation as:

$$
\begin{aligned}
& \qquad s_{n}=\frac{1}{N} \sum_{k=0}^{2 N-1} d_{k} \exp (j 2 \pi k n / N) \\
& \text { where } n=0,1, \cdots, 2 N-1 .
\end{aligned}
$$

[Insert Figure 1]

For the receiver, OFDM demodulation is performed as:

$$
\begin{aligned}
& d_{k}=\frac{1}{N} \sum_{n=0}^{2} s_{n} \exp (j 2 \pi k n / N) \\
& \text { after differential decoding, original bits can be obtained as: } \\
& d_{k, n}=\frac{d k, n}{d k-1, n}
\end{aligned}
$$

A block chart of the OFDM modular is shown in Fig 2.

\subsection{Mode selection protocol}

To achieve flexibility under different channels, the mode switch of the DM modem system will be controlled by a mode selection protocol according to the quality of channel. Under the proposed protocol, a terminal initializing the data transfer will perform handshaking in advance of any data communication.

To guarantee the reliability of the handshaking transfer, DSSS is used in the handshaking stage. The main purpose of the handshaking stage is to obtain the quality of the channel to determine the mode to be used for data communication. Specifically, the handshaking protocol 
consists of the following procedure:

[Insert Figure 2]

(1) Terminal A (which is the one initializing the transfer) sends a request frame to Terminal B (which is the target terminal of transfer) to ask for a channel measurement. The request frame contains a PN signal acting as a channel probe.

(2) Upon receiving the request frame, Terminal B performs channel measurement via matched filtering of the PN signal.

(3) Terminal B sends a clear frame back to Terminal A, which contains the channel parameters obtained by the measurement.

(4) After receiving the clear frame and acquiring the channel parameters, Terminal A selects the suitable modulation mode (DSSS or OFDM) according to the channel parameters and mode selection mechanism and then sends a mode confirm frame to set the mode of Terminal B.

(5) After receiving the mode confirm frame and extracting the specific mode contained, Terminal B send an acknowledge frame back to Terminal A.

(6) Terminal A and B enter the data communication stage with the selected modulation mode after Terminal A receives the acknowledge frame.

Considering the severity of UWA channels, the control frames transferred during the handshaking stage will be transmitted again if the corresponding response is not received after a specific time. The design of the handshaking protocol ensures the suitable modulation mode will be selected to accommodate the specific channel each time any terminal initializes data transfer.

\section{SYSTEM IMPLEMENTATION}

The UWA communication testbed used to demonstrate the dual mode scheme was built based on a TI TMS320C6713 DSP. The dual mode testbed consists of a DSP chip acting as the 
processing core of the testbed, a DAC and ADC for data output and acquisition, an RS232 serial port, a power amplifier, preamplifier, and two transducers (as shown in Fig.3). The DSSS and OFDM modulation schemes as well as the mode selection protocol are implemented on the same DSP.

\section{[Insert Figure 3]}

[Insert Table 1]

The modulation parameters for the OFDM and DSSS modulars are presented in Table 1 and Table 2 Respectively. The bandwidth of the transducer is $13-18 \mathrm{k} \mathrm{Hz}$, with the sampling rate at 96ksps.

The probe used for channel measurement is a 127-chip PN signal with a width of $31.75 \mathrm{~ms}$. After the matched filtering process, the correlation peak and the background noise of the received signal corresponding to the channel probe is obtained to calculate the SNR of the current channel. In the proposed system, SNR is used as the criterion in the mode selection protocol to select the suitable modulation mode, specifically, when SNR is higher than 10dB, the OFDM mode will be selected to achieve high data rate, otherwise, the system will work in the DSSS mode to ensure reliability. As the main purpose of this work is to test the dual mode strategy, no error coding technique is adopted in the proposed testbed.

The experiment in the ocean was carried out at Wuyuan Bay, Xiamen, China. The depth of the experiment area is approximately $7 \mathrm{~m}$ under the pier and $12 \mathrm{~m}$ offshore. The arrangement of the experiment is shown in Fig.4. The transducer of the transmitter was suspended from the pier at a depth of $5 \mathrm{~m}$ from the sea surface. The transducer of the receiver was suspended from a boat at a depth of $5 \mathrm{~m}$ from the sea surface. Under the same transmission power level, signals were sent over distances of $300 \mathrm{~m}$ and $2 \mathrm{~km}$ to test the performance of the testbed system under 
different SNR. To simulate different kind of communication missions, a 16 gray level image was transmitted in OFDM mode and ASCII code characters were sent in DSSS mode.

\section{[Insert Table 2]}

[Insert Figure 4]

\section{EXPERIMENTAL RESULTS}

Firstly, the DM modem testbeds were tested at the distance of 300m. During the DSSS mode handshaking, the matched filtering output of the channel probe is shown in Fig.5(a), which indicates an SNR of 15dB. According to the SNR criterion defined in the mode selection protocol, the OFDM modulation mode is selected for data communication. The OFDM signals received are shown in Fig.5(b). The final image obtained at a bit rate of 6.8kbps is presented in Fig.6(b), which corresponds to a bit error rate of 3.5\%.

At the distance of 2km, the correlation curve of the channel probe (shown in Fig.7(a)) reveals an SNR of $5 \mathrm{~dB}$. As a result, the low data rate DSSS mode is selected by the mode selection mechanism. In Fig7.(b), we can see the DSSS waveform received at 2km which contains some serious impulsive noise. The DSSS mode demodulation achieves a bit error rate of $0.5 \%$ with a low data rate of $63.45 \mathrm{bps}$. These preliminary results of the sea trial show that, the dual mode mechanism enables the proposed UWA modem testbed to achieve good performance under different channel conditions, with low rate DSSS for low SNR and high rate

[Insert Figure 5, 6, \& 7]

\section{CONCLUSION}

In this paper, we have presented the R\&D of a Dual-mode modem testbed, in which DSSS and OFDM are selected as the candidate modulation schemes of the DM system to achieve 
flexibility under different channel characteristics. The design of DSSS/OFDM modulars, modulation selection protocol and system configuration were introduced.

The UWA modem testbed system is implemented based on a TI DSP platform. Considering the implementation complexity, classic DSSS/OFDM modulation modulars as well as relatively simple SNR based mode selection protocol are adopted to fulfill the functional demonstration of the proposed dual mode system. Finally, sea trials at Wuyuan bay are performed to test the effectiveness of the proposed system. Experimental results under different SNR validate the improved channel adaptability of the dual mode system, which has the potential of being developed and applied in related fields of underwater applications.

\section{ACKNOWLEDGMENT}

This material is based upon work partially supported by Nature Science Foundation of China (NSFC)

(No. 10704063) and “NCET” Foundation of Fujian Province.

\section{REFERENCES}

[1] Liao, D Z; Harrold, S O; Yeung, L F, “An underwater Acoustic Data Link for Autonomous Underwater Vehicles”, IEEE Int. Conf. in Circuit and Systems, p28-33, Singopare, July 1995.

[2] Bridget Benson, Ali Irturk, Junguk Cho and Ryan Kastner, "Energy Benefits of Reconfigurable Hardware for Use in Underwater Sensor Nets", IEEE Reconfigurable Architectures Workshop , May 2009

[3] D.B.Kilfoyle, A. B. Baggeroer, “The State of art in underwater acoustic telemetry”, IEEE J.Oceanic. Eng., Vol, 25(1), pp.4-24, 2000

[4] M.D.Green, J.A.Rice, "Channel-Tolerant FH-MFSK Acoustic Signaling for Undersea Communications and Networks”, IEEE J.Oceanic. Eng., Vol, 25(1), pp.28-39, 2000

[5] M. Jian, W. H. Yung, B. Songrong "An Efficient IF Architecture for Dual-Mode GSM/WCDMA Receiver of a Software Radio", IEEE International Workshop on Mobile Multimedia Communications, San Diego, USA, November 1999.

[6] L. Freitag et al., "The WHOI Micro-Modem: An Acoustic Communications and Navigation System for Multiple Platforms," Proc. IEEE Oceans Conf., 2005

[7] M.Stojanovic, L.Freitag, "Acquisition of Direct Sequence Spread Spectrum Acoustic Communication Signals”, Oceans 2003 Proceedings, Vol. 1, pp.22-26, Sep. 2003

[8] E.M.Sozer, J.G..Proakis, M. Stojanovic, J.A.Rice, A.Benson, M.Hatch, “Direct sequence spread spectrum based modem for underwater acoustic communication and channel measurements", Oceans'99, MTS/IEEE, Riding the Crest into the $21^{\text {st }}$ Century, Vol.1,pp. 228-233. 1999 
[9] S. Coatlelan and A. Glavieus, "Design and test of a coded OFDM system on the shallow water acoustic channel”, in Proc. OCEANS Conf., Sep. 1994, pp2065-2070

[10] M. Stojanovic, "Low complexity OFDM detector for underwater acoustic channels", in Proc. MTS/IEEE OCEANS conf., Boston, MA, Sep. 18-21, 2006

[11] B.Li, S.Zhou, M. Stojanovic and L.Freitag, "Pilot-tone based ZP-OFDM demodulation for an underwater acoustic channel”, in Proc. MTS/IEEE OCEANS conf., Boston, MA, Sep. 18-21, 2006 OFDM for high SNR. 

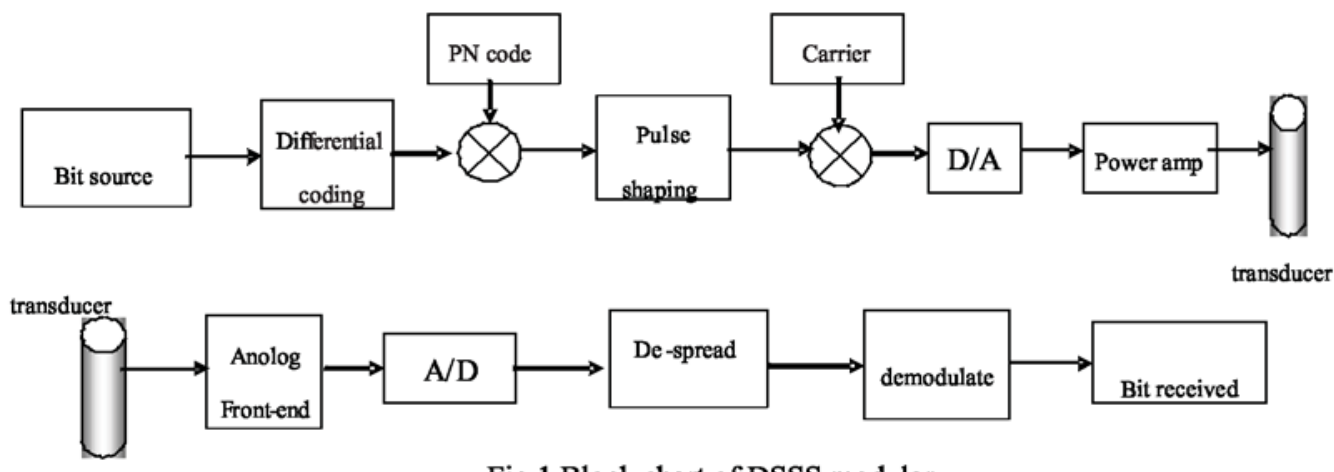

Fig.1 Block chart of DSSS modular

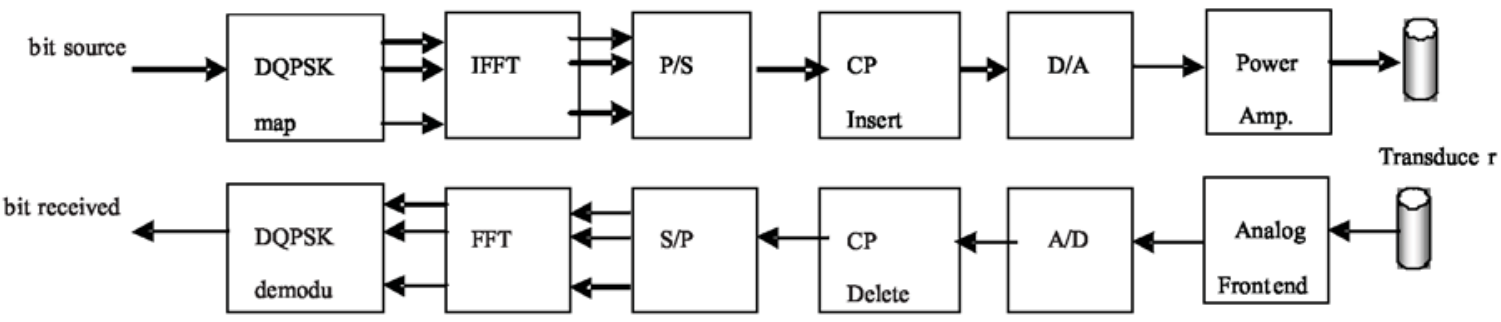

Fig.2 Block chart of OFDM modular

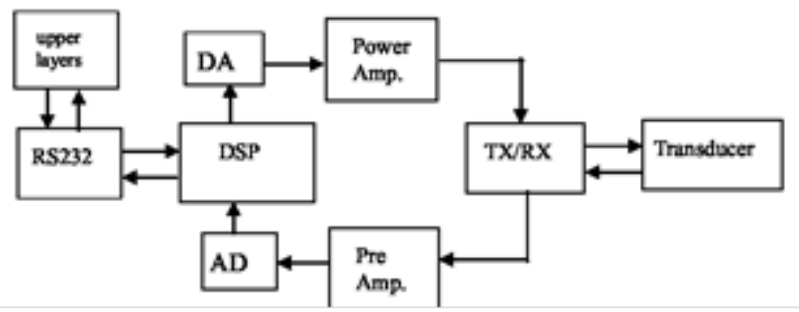

Fig .3 Bock chart of the testbed 


\begin{tabular}{|l|c|l|r|} 
FFT length & 4096 & Subcarrier space & $23.4 \mathrm{~Hz}$ \\
\hline Symbol duration & $42.67 \mathrm{~ms}$ & BW oceupied rate & $93.5 \%$ \\
\hline Bandwidth & $2.08 \mathrm{k}$ & Bit per frame & 4000 \\
\hline Guard time & $10.67 \mathrm{~ms}$ & Length of reference & $53.4 \mathrm{~ms}$ \\
\hline Frame length & $534 \mathrm{~ms}$ & Bit rate & $6.8 \mathrm{kbps}$ \\
\hline Frame period & $587.4 \mathrm{~ms}$ & Modulation & DQPSK \\
\hline
\end{tabular}

Table.1 Parameters of OFDM modulation

Table.2 Parameters of DSSS modulation

\begin{tabular}{|l|c|l|c|}
\hline Spread gain & 63 & Guard time & $20 \mathrm{~ms}$ \\
\hline Carrier frequency & $16 \mathrm{kHz}$ & Bit per frame & 192 \\
\hline Chip rate & $4 \mathrm{k}$ & PN code & M sequence \\
\hline Bit rate & 63.49 & Modulation & DBPSK \\
\hline
\end{tabular}

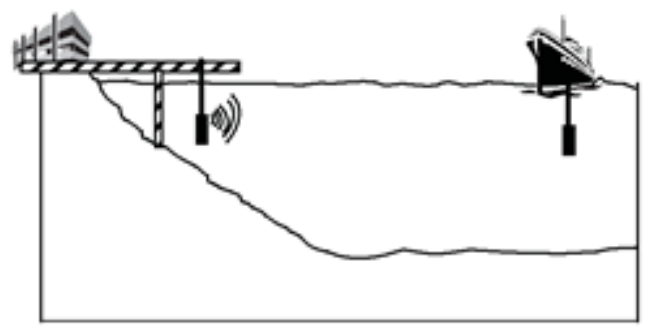

Fig.4 Sea experiment configuration 


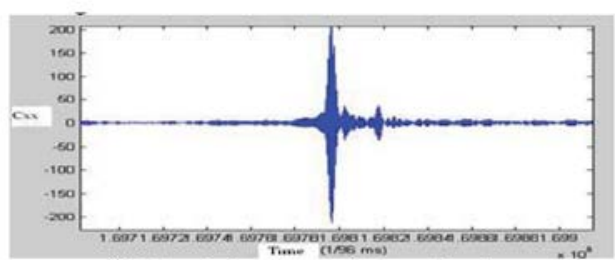

(a) Correlation curve of channel probe

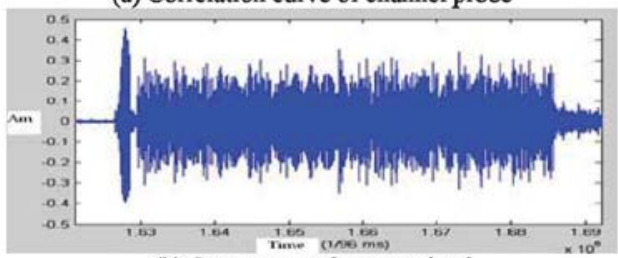

(b) OFDM waveform received Fig.5 Signals received at $300 \mathrm{~m}$
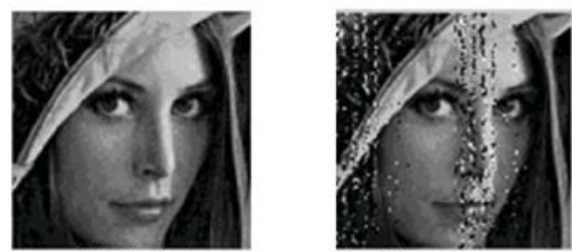

(a) Original

(b) Received

Fig.6 Image received at $300 \mathrm{~m}$

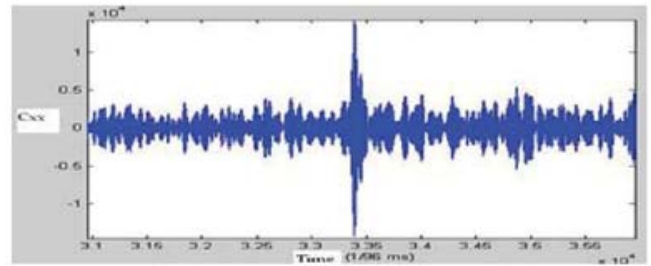

(a) Correlation curve of channel probe

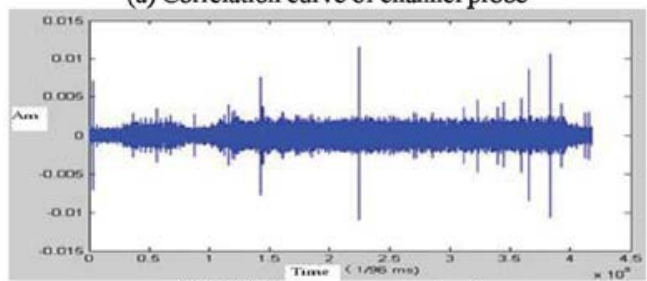

(b) DSSS waveform received

Fig.7 Signals received at $2 \mathrm{~km}$ 